\title{
In situ Sintering of Agglomerated 3\% Yttria-stablized Zirconia
}

\author{
Hasti Majidi $^{1}$, Troy B Holland ${ }^{2}$ and Klaus van Benthem ${ }^{1}$ \\ 1. Department of Chemical Engineering and Materials Science, University of California, Davis, CA, \\ 95616, USA \\ 2. Department of Mechanical Engineering, Colorado State University, Fort Collins, CO, 80523, USA
}

We report in situ transmission electron microscopy (TEM) observations and quantitative shrinkage analysis of agglomerated 3\% yttria-stablized zirconia (3YSZ) during sintering. Unlike post-mortem characterizations, in situ heating in TEM enables direct observations of the particle agglomerates during sintering and thus is capable of obtaining mechanistic insight unambiguously. Asoro et al. have used HAADF STEM in situ observations to monitor a two-particle model system of platinum nanoparticles and revealed surface diffusivity during initial stage of sintering.[1] Initial stage of sintering (neck formation and growth) only depicts particle fusion and elongation. To observe the actual densification during sintering, the TEM sample of interest should contain at least 3-4 particles to form a pore. Hence, we chose a particle agglomerate of 3 YSZ to expand the study of neck formation and growth further and investigate pore shape and shrinkage during in situ sintering.

Time-resolved observations were obtained utilizing TEM, STEM and EELS while performing the sintering in the microscope by employing the Protochips Aduro TEM heating holder. Direct TEM observations while exposing particle agglomerates to elevated temperatures provide new information on morphological and structural changes, formation of necks and pore closure during sintering. Figure 1 shows real-time observations of a 3 YSZ agglomerate upon in situ sintering. The blue arrows highlight two particles that have no contact at $25{ }^{\circ} \mathrm{C}$, Figure 1 (a), form a neck at $1023{ }^{\circ} \mathrm{C}$, Figure 1(b). The neck formation is followed by neck growth at $1160^{\circ} \mathrm{C}$, Figure 1 (c). In addition, red arrows in Figure 1 show the size reduction in pores. Some pores are completely eliminated due to densification. Moreover, consolidation of 3 YSZ coincides with Oswald ripening. Green arrows in Figure 1 indicate particle coalescence. Three particles at room temperature fuse together and form one bigger particle at $1160{ }^{\circ} \mathrm{C}$.

We combined in situ sintering with an analysis methodology to evaluate the agglomerate shrinkage as a function of temperature. To our knowledge, this is the first real-time TEM observation that was used to generate consolidation curves for 3YSZ agglomerates. We developed a MATLAB image processing tool and calculated the projected agglomerate area with and without internal pores. The projected agglomerate area is called dark area when the internal pores are excluded and closed area when it includes pores. Figure 2 shows both dark area and closed area shrinkage curves for the 3 YSZ agglomerate. Sintering onset temperature is determined to be $\sim 950{ }^{\circ} \mathrm{C}$.

Consolidation is a result of pore elimination. Hence, the projected area of the agglomerate without internal pores (dark area) is not expected to reduce in size during sintering. However, Figure 1 shows that dark area is shrinking with the same densification trend as the closed area. We hypothesize that the size reduction in the agglomerate area even when the internal pores are not included is due to the limitations in TEM imaging. TEM imaging of a 3-D object is limited to two dimensions and is incapable of providing information in the third dimension. We therefore used in situ STEM-EELS analysis to generate mass-thickness maps of the agglomerate as a function of temperature. EELS analysis disclosed that the average mass thickness of the agglomerate increases during in situ sintering. This is likely 
because of the encapsulated pores in the third dimension. Those type of pores would be hidden to electron beam and TEM 2-D imaging is unable to recognize them. Hence, filling those pores and also material's sliding and twisting make some visible particles in the boundaries hide behind inner particles and lead to shrinkage in dark area of the agglomerate.

The technique opens up a new direction to easily connect the microstructural evolution to densification behavior during processing. We are currently employing this technique to $3 \mathrm{YSZ}$ agglomerates while exposing them to electrostatic fields during in situ heating experiments. The goal is to identify mechanisms that enhance densification due to applied electric fields or currents.

\section{References:}

[1] M A Asoro et al, Nanotechnology (2010) p. 025701.

[2] This research was funded by the Army Research Office (Program manager Dr. Suveen Mathaudu) under award \#W911NF1210491.
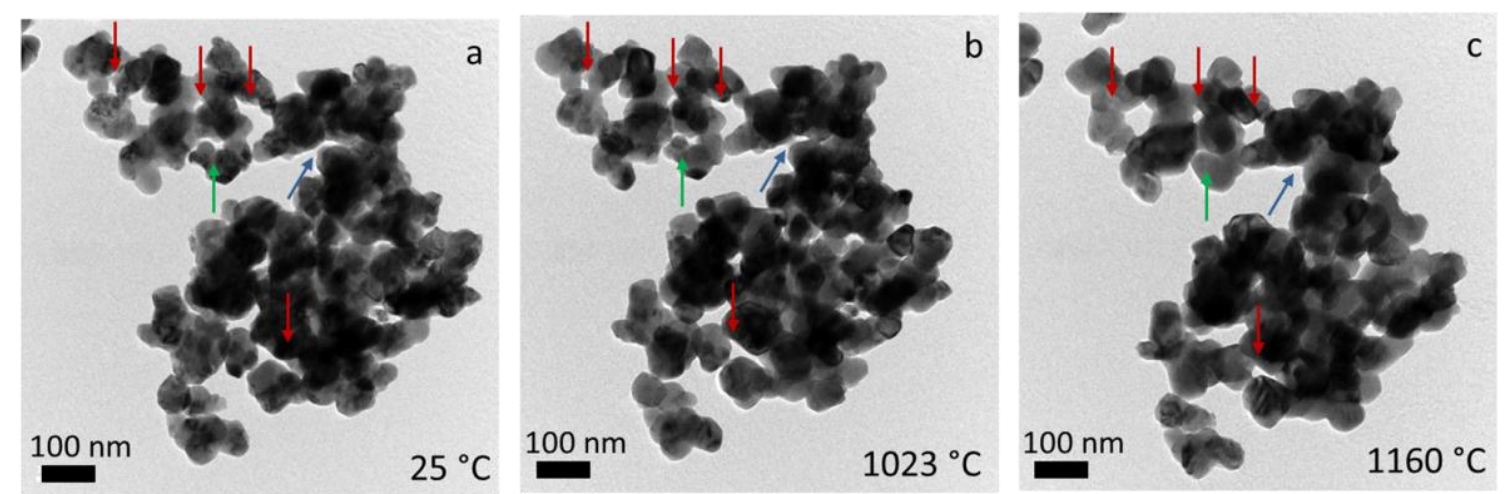

Figure 1. Real-time TEM micrographs of a $3 \mathrm{YSZ}$ agglomerate (a) before and after in situ heating at (b) $1023{ }^{\circ} \mathrm{C}$ and (c) $1160{ }^{\circ} \mathrm{C}$. Blue arrows indicate neck formation and growth, and red arrows highlight pore shrinkage. Also, green arrows show particle fusion during in situ sintering.

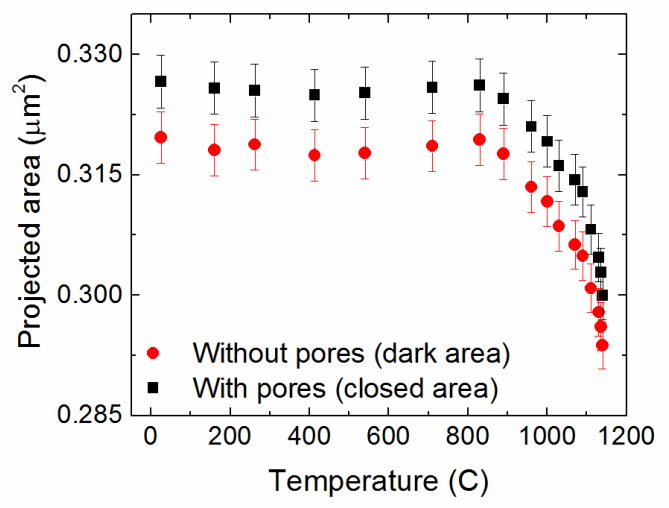

Figure 2. Calculated projected areas of an in situ sintered $3 \mathrm{YSZ}$ agglomerate. 